\title{
Profesores de tiempo bailable: violencia institucional y falta de seguridad laboral para los docentes
}

\section{Ser profesor de tiempo variable era ser cool}

Hasta ahora, a lo largo de este trabajo, se han abordado las diferentes manifestaciones de la violencia escolar, desde los más diversos entornos socioculturales: el punto de vista de los alumnos, de los profesores que han sido violentados, en diferentes contextos sociales de México y en muy variados estratos de la sociedad mexicana.

Ahora el turno es para hablar de la situación laboral, financiera y legal con la que cuentan gran cantidad de profesores en el país, sobre todo en educación media y superior, pero también en educación básica, principalmente en las escuelas particulares. Carecen, en una gran cantidad de ocasiones, de las más mínimas prestaciones y seguridades laborales.

A finales de los años ochenta e inicios de los años noventa, en las universidades privadas en México, principalmente en el ala de las escuelas superiores que en una época administraba la congregación de los jesuitas, surgió la categoría administrativa de los profesores de tiempo variable. Lo que significaba que no tenían que estar todo el tiempo al interior de la institución, asistiendo únicamente en los horarios que se 
les asignaron previamente o que, al inicio, ellos mismos elegían para impartir sus materias.

De las universidades privadas de la Compañía de Jesús, esta categoría fue extendiéndose hacia otros niveles educativos inferiores: bachilleratos, secundarias y primarias. En pocos años, fue migrando hacia otras instituciones privadas, hasta generalizarse casi a un $100 \%$ en todo el país, e incluso comenzar a ser adoptado lenta y discretamente por las instituciones públicas. Con otro nombre, pero muy similar en su esencia.

Sugerimos que, en una "época”, estas escuelas fueron "administradas” por los jesuitas y la Compañía de Jesús, porque durante varias décadas los religiosos sí tuvieron bastante incidencia en los planes de estudio y métodos educativos que se aplicaban en sus colegios y universidades. Aunque, luego, estas mismas instituciones fueron entregadas a la iniciativa privada y a asociaciones de empresarios, inversionistas privados y banqueros, quienes en la actualidad administran estas casas de estudio con objetivos y finalidades meramente mercantilistas y empresariales. Por ello, se queda fuera la figura de los jesuitas como meros accesorios decorativos, a lo mucho, o como parte de una imagen con la que se pretende vender el concepto de una educación liberal cristiana a un costo sumamente elevado, al punto de resultar descaradamente alto. Es una educación liberal con la que en la actualidad muy poco tienen que ver las escuelas y universidades que alguna vez administró la Compañía de Jesús. Bastante alejadas ahora de los ideales cristianos ignacianos que otrora les inspiraron.

A tal grado que, por ejemplo, actualmente los cobros de colegiaturas del Iteso, en la ciudad de Guadalajara y otros colegios, los cuales se pagan con bastantes dificultades, indican estar inspirados en la pedagogía jesuita y son administrados por el fideicomiso del grupo Bancomer.

En los años ochenta, para tratar sus adeudos de colegiaturas, los alumnos y padres de familia se dirigían directamente con el administrador del colegio o la universidad, o el secretario del rector, quienes, por lo general, eran sacerdotes, religiosos pertenecientes a la Compañía de Jesús. Los adeudos de las colegiaturas y pagos se negociaban con estas figuras, quienes realmente aplicaban los ideales ignacianos en sus decisiones financieras, eran bastante humanos en sus juicios y 
decisiones. Después, cuando las finanzas de estas instituciones fueron entregadas al fideicomiso bancario español, quien por cierto posee un siniestro pasado de corrupción y crimen en el Viejo Continente, todo cambió drásticamente. A partir de entonces, los estudiantes y padres de familia tuvieron que vérselas con una institución a la que en lo más mínimo le interesaban sus historias personales para negociar sus deudas. Esta estaba encargada de cobrar a sus deudores a toda costa, haciendo acopio de las propiedades de las familias de estudiantes, e incluso embargándoselas si no podían cubrir sus pagos.

Desde los años setenta, los jesuitas tuvieron sus momentos de vanguardia con respecto a la educación en México, en comparación con otras instituciones educativas que les competían. La Compañía de Jesús tuvo sus instantes en que realmente innovó en México y estuvo a la cabeza de la educación en nuestro país; particularmente, con su propuesta de educación personalizada, derivada de las enseñanzas y filosofía de San Ignacio de Loyola que pretendía educar líderes independientes y librepensadores, al igual que los discípulos casi milicianos de San Ignacio: sus guerreros de la contrarreforma; estos últimos desarrollaron plenamente su potencial intelectual y emocional para integrarse a las sociedades. En los noventa, la educación jesuita logró colocarse incluso por encima de la que sustentaban los colegios maristas, conocidos en México como colegios Cervantes. Estos lograban altos índices en sus pruebas de gramática, álgebra, aritmética y cálculo, pero los jesuitas tenían la particularidad de ayudar a sus estudiantes a cultivar la amistad, el trabajo en grupo, la solidaridad, la resolución de conflictos, la conciencia social, la innovación, la creatividad y el liderazgo.

De las filas de sus instituciones, en los ochenta y noventa, egresaron importantes personajes de la ciencia, la cultura, la política y el arte. Personalidades de la talla del investigador Fernando Leal Carretero, el cineasta Guillermo del Toro, el político independiente Pedro Kumamoto, incluso, se dice, el subcomandante Marcos, junto con un sinnúmero de empresarios, políticos, líderes sociales y artistas.

Hoy en día, los colegios maristas destronaron en todas esas áreas a los jesuitas. Y la situación académica es completamente opuesta; perdieron las escuelas de la Compañía de Jesús no solo su nivel académico, 
sino aquellos valores tan importantes que pregonaba y aplicaba en épocas ya lejanas y perdidas en el tiempo.

Los grupos de banqueros y empresarios, quienes realmente fueron los dueños desde siempre de la educación jesuita, comenzaron a reclamar su derecho innegable de controlar y prescribir las directrices de la educación de sus colegios y universidades. Entonces, a finales de los ochenta e inicios de la década de los noventa, los jesuitas, quienes estuvieron a la cabeza de dichas instituciones por años, comenzaron a ser relegados paulatinamente. Incluso, fueron expulsados casi por completo de las mencionadas escuelas, aunque esto no se revela en la mercadotecnia de estas, debido a que siguen insistiendo en que su educación es de corte jesuita.

En estas décadas, creció a niveles escandalosos la cantidad de ofertas educativas, a costos mucho más bajos en comparación con las escuelas y colegios jesuitas. Las instituciones que administraba la Compañía de Jesús comenzaron a perder grandes cantidades de estudiantes y clientes, quienes buscaban otras opciones educativas mucho más económicas y casi de la misma calidad, e incluso mejores. Las universidades públicas también crecieron considerablemente, formando redes universitarias en casi todos los Estados de la República mexicana. Captaron a bastantes estudiantes que alguna vez estudiaron en escuelas jesuitas o que trataron de hacerlo, pero no consiguieron finalizar sus estudios, debido a los altos e impensables costos.

Todo esto fue dándose en el contexto de una serie de reformas educativas en México, orquestadas por los Gobiernos neoliberales desde inicios de los años noventa que le fueron dando cada vez más a la educación en todo el país un carácter técnico, pragmático y empresarial.

En las universidades de corte jesuita, como el Iteso y la Universidad Iberoamericana, surgió entonces la categoría de profesor de tiempo variable, en contraposición a los profesores de tiempo completo, que cada vez son menos. Los tiempos completos gozan de todas las prestaciones de ley, les son pagadas sus vacaciones y tienen derecho a una jubilación. Si son despedidos, las empresas-escuelas están obligadas a indemnizarlos económicamente.

Inicialmente, este concepto de tiempo variable tenía como única y legítima finalidad captar los perfiles docentes de profesionales liberales 
que trabajaban directamente en sus campos del conocimiento, y buscaban que el personal que continuara en sus áreas laborales impartiera al mismo tiempo una o dos materias, nada más.

Se trataba de que estos profesionales transmitieran a sus alumnos su experiencia única en los diversos campos de trabajo, y de acercar a las universidades y bachilleratos las experiencias prácticas de los expertos, quienes se desempeñaban en las diversas áreas del conocimiento, y no centrarse en personal docente e investigadores que se dedicaran exclusivamente a impartir clases y que, incluso, carecieran por completo de la experiencia laboral fuera de las escuelas: en la vida real.

Digamos que los pedagogos jesuitas se dieron cuenta que entre su personal predominaba de manera preocupante el perfil de profesores que jamás habían ejercido su profesión, además de dar clases y permanecer toda su vida en el ámbito educativo, primero como alumnos y luego como docentes. Entonces, la iniciativa de los profesores de tiempo variable tuvo algunos motivos interesantes en sus inicios.

Esta noción resultó bastante propositiva al comienzo, pues bastantes profesionistas, quienes ejercían directamente en el ámbito clínico, empresarial, cultural, legal, tecnológico, artístico, etc., se integraron a las escuelas jesuíticas para colaborar y compartir sus experiencias y conocimientos con los jóvenes estudiantes.

Algo que no se tuvo en cuenta, por lo menos no conscientemente, era que las filas de profesores de tiempo variable aumentaran enorme y obscenamente en las últimas décadas; particularmente, debido al desempleo en México y a la falta de oportunidades laborales para millones de profesionistas y egresados de tantas diferentes universidades. Entonces, una categoría como los tiempos variables, que tenía como supuesta finalidad contratar y reclutar a profesores que compaginaran la impartición de una o dos materias, con su trabajo profesional en su campo específico de trabajo, se pervirtió por completo. Se comenzó a utilizar como instrumento legal y fiscal para mantener a una inmensa población de docentes o personas que se dedicaban a la enseñanza, quienes trabajaban bajo un costo bajísimo, sin prestaciones laborales ningunas ni posibilidad de construir algún proyecto de vida. Trabajaban a destajo y cobraban únicamente por hora pagada. 
La cantidad de profesores que trabajan por hora es cada vez mayor en México. La inmensa mayoría ya solo se dedica exclusivamente a dar clases y no puede laborar en otras áreas, más que con contadas excepciones. Esto debido a las grandes exigencias administrativas y curriculares, de las que se hablará enseguida, y también a los bajos costos por hora clase impartida, lo que los obliga a tener que dar más clases si es que quieren obtener un ingreso suficiente para sobrevivir.

Asimismo, afectando la tranquilidad financiera y social de bastantes docentes de todo México, el esquema de profesores de tiempo variable fue adoptado y generalizado prácticamente por todas las universidades privadas o particulares, y colegios de todos los niveles educativos en México.

Lo anterior dio como resultado una aplastante plantilla docente en el país que no cuenta absolutamente con ningún tipo de seguridad laboral ni ningún tipo de prestación social, que no recibe sueldo durante las vacaciones y percibe un irrisorio pago solo por hora trabajada.

Tenemos noticia de que aún existen instituciones de educación media y superior de carácter privado —y aún más, también públicas- que pagan a sus docentes el irrisorio costo de 28 pesos la hora clase. Y de ahí hacia arriba, un poco solamente, oscilando desde los 30 pesos, hasta los 60, 80, 100 y 150 pesos por hora. De cualquier manera, los pagos resultan muy bajos teniendo en cuenta el enorme trabajo real de estudio, planeación e investigación presente detrás de cada hora clase dada. Los administradores de las instituciones educativas no tienen esto en cuenta.

Con las más recientes reformas educativas, a partir del año 2000, se le imprimió a la educación en México un carácter aún más empresarial y técnico; se implementó, desde el ámbito laboral, la noción de certificación, lo cual conlleva la utilización de formatos para llenar y dar cuenta de cada proceso institucional. Los formatos han llegado de tal modo a formar parte de la vida de los docentes y trabajadores de la educación que una inmensa cantidad de tiempo se invierte en su realización, incluso mayor a lo que permanecen frente a grupo.

En el caso de los profesores de tiempo variable, estos también tienen que elaborar una inmensa cantidad de formatos de planeaciones, evidencias grupales, etc., sin embargo, como a ellos no se les paga más 
que su hora clase una vez que fue impartida, la labor de planeación consiste en una monótona e interminable actividad que deben desarrollar fuera de clase, hasta en sus casas, sin ninguna remuneración. Si por algo, el docente se inconformara con estas obligaciones por las que no recibirá compensación económica, siempre habrá otros muchos profesionales dispuestos a tomar sus horas para trabajar casi gratuitamente.

En la década de los noventa, todavía en el Iteso y la Ibero de los jesuitas, se enorgullecían algunos profesores de pertenecer a la categoría de tiempos variables. Algunos lograban aún compaginarlo con otras actividades independientes, como las consultorías, la psicoterapia, el freelance, como suele decirse, que les proporcionaban ingresos extras y complementarios.

Algunos se ufanaban de pertenecer a las filas de docentes del Iteso, y vendían una imagen de profesores hipsters: comunicólogos, pedagogos, diseñadores gráficos, ingenieros industriales, arquitectos, quienes llegaban a la universidad a impartir sus clases en bicicleta, moto, vestidos con bermudas, ataviados con sus barbas prolongadas, cabello largo, algunos con arracadas y guaraches. Se decía que los profesores, junto con algunos de los alumnos, se fumaban el pasto de sus jardines, lo que aludía a que eran bastante desenfadados y hippis. Digamos que en aquella década, antes de la entrada del año 2000, pertenecer a las filas de profesores de tiempo variable del Iteso, la Ibero, la Univa, etc., era considerarse a sí mismo con cierto e importante estatus social. Ser profe del Iteso era ser cool.

Como se verá a continuación, esta imagen y las ideas de estatus social asociadas a ella han cambiado radicalmente en menos de diez años. Ser profesor tanto de las universidades jesuitas, como casi de cualquier otra, equivale en la actualidad a no tener el respeto de los alumnos, los padres de familia, ni el respaldo alguno de su propia institución.

\section{Profesores de tiempo bailable}

En el Iteso, la Universidad Jesuita de Guadalajara, los profesores de tiempo variable bromean entre ellos, con cierta discreción, cuidando de no ser escuchados por los directivos. Se dicen a sí mismos ser más bien de "tiempo bailable". La institución los ha bailado, los hace bailar 
y proseguirá haciéndolo mientras exista o ellos laboren ahí. Estos docentes bailan al ritmo que les manden porque tienen que adaptarse a los horarios que les son impuestos por parte de sus coordinadores y directivos. Bailan porque también pueden ser echados en cualquier momento con el lujo de que incluso no se les avise el haberse prescindido de su trabajo. Las historias tristes de algunos profesores que llegan a su salón de clase y encuentran a otra persona al frente de grupo, sin que nadie les haya enviado siquiera un correo electrónico de aviso previo, son numerosas y conocidas.

Originalmente, la concepción del tiempo variable permitía al docente acomodar sus horarios para hacerlos compatibles con el resto de las actividades laborales que desempeñaban; pero eso fue en los años ochenta. Desde hace décadas, en el Iteso, los horarios se organizan de manera interdepartamental. Lo que en pocas palabras quiere decir que los profesores no serán tomados en cuenta en lo absoluto al decidir los acomodos semestrales de los horarios de clase.

Más bien son los docentes, si es que quieren trabajar ahí, los que deberán acomodarse a los horarios como les sean impuestos. Lo que de entrada ya les pone difícil la posibilidad de compaginar sus clases con otro tipo de actividad, o siquiera con la impartición de materias en otra escuela. Lo que contradice por completo la concepción inicial del tiempo variable.

Los coordinadores les dicen que los horarios se acomodan de acuerdo a la organización de los planes de estudios y a la oferta que cada departamento realiza. Pero la realidad es que la institución responde en función de las demandas de los estudiantes, los que acomodan sus horarios, eligen las materias y seleccionan a los profesores más populares. Hace muchos años que el estudiantado tiene poder absoluto para quitar y poner a los profesores que desee. Y los profesores caminan cada día sobre una cuerda floja bastante riesgosa. Cualquier cosa puede constituirse en el más mínimo pretexto para resultar mal evaluados en el fin de curso, y ser echados para siempre del trabajo.

Tenemos la noticia de una veterana profesora de psicología, quien resultó muy mal evaluada al finalizar el semestre por parte de los estudiantes, debido a que a las alumnas no les gustaba el olor de su perfume. “¿Es cierto que no me vas a dar más clases porque no les gustaba 
mi perfume..."?, preguntó a su coordinador, encargado de asignarle materias. La profesora no volvió nunca más a ser contratada por la universidad, aunque tenía veinte años dando clases y el coordinador fue su alumno cuando este era estudiante.

El caso de Gamaliel, profesor de tiempo variable en las carreras de Arquitectura, Comunicación y Diseño, revela muchas y muy diversas experiencias en sus casi ocho años impartiendo clases en esta institución. El Iteso es su alma máter, pues egresó de ella en 1999, pero él es doblemente jesuita porque desde la primaria estudió en el Instituto de Ciencias, uno de los colegios más prestigiosos de la Compañía de Jesús en Guadalajara:

Yo estuve desde el kínder en el Ciencias, desde maternal, con una maestra que se llama Gaby, ya se jubiló. Estudié todos los grados escolares en el colegio, pero a mitad de la prepa falleció mi papá. Me faltaba un año exactito para acabar la escuela y creí que no iba a poder seguir estudiando más. Un año antes mis papás se divorciaron, y mi papá se había ido a vivir a Tapalpa. Así es que se me juntaron un montón de cosas emocionales, financieras y familiares. La época de finales de la preparatoria y mediados de la universidad ha sido una de las más duras de mi vida.

Pensé que no iba a haber educación universitaria para mí, se me cerró el mundo. Yo era el más chico de mis hermanos, ya todos se habían casado y tenían su carrera universitaria. No más faltaba yo. Después supe que mi papá había dejado un financiamiento en Banamex para que se cubriera la mayor parte de mis estudios. Yo quise mucho a mi papá, hasta que no se murió supe muchas cosas y aprendí más de él, de cosas que hizo, pero que nadie de la familia sabíamos.

De manera que al entrar al Iteso lo hice con muchas ganas, entré a la carrera de Arquitectura, que es de las más caras en esa universidad. Aproveché todas las materias, también leí muchísimo. Desde entonces me encantaba la literatura, las novelas y los cuentos, también los libros sobre ciencias, medicina, matemáticas, física cuántica, esoterismo. Me la vivía en el Iteso, prácticamente 
vivía ahí. Si no estaba en mis clases, estaba haciendo la tarea, y si no, estaba en la biblioteca, o en los jardines acostado, siempre leyendo. No tuve novia hasta que acabé la carrera.

Casi al final de la carrera, aunque me costó mucho trabajo reconocerlo, me di cuenta de que la arquitectura no era lo mío. Me dio muchísima tristeza, con todo el trabajo que le dio a mi papá dejar el dinero para financiar la licenciatura y el esfuerzo de mi mamá que también me ayudaba y de mis hermanos, que me echaban también la mano. No quería decepcionarlos. Me dio una depresión bien fuerte, casi me voy al manicomio; se me habían juntado un montón de cosas emocionales: el divorcio de mis papás, la muerte de mi papá, la carrera, la situación financiera. Mi mamá se fue a vivir a Torreón con sus hermanos. Luego se volvió a casar. Mis hermanos todos ya habían hecho su vida, mi hermana Tere, la que era más cercana a mí se fue a vivir a Colombia con su esposo. En el sexto semestre de la carrera me encontraba completamente solo y así me sentía.

Entonces recuperé la cordura, tomé la decisión de acabar la carrera como fuera. ¿De algo me iba a servir, no...? No me iba a dejar hundir por la depresión y la desesperanza, iba a salir adelante.

Desde el inicio me vinculé con despachos de diseño gráfico y de mercadotecnia. La arquitectura me dio tablas para eso. Aunque jamás la he ejercido directamente. Tuve muy buena formación en el Iteso, la escuela de arquitectura del Iteso es de las mejores del país.

Trabajé desde el año 2000 al 2005 en Boston, en un despacho de diseño gráfico y publicidad. Escribía para una revista sobre artes y arquitectura y diseñaba los gráficos para un montón de empresas, también hacía páginas web. En el tiempo libre me la pasaba en la biblioteca de la Universidad. Fue una época muy desahogada económicamente, la verdad la extraño bastante. No creas, ahora como profesor ni en sueños ganas lo que pagan las grandes firmas de publicidad. Pero bueno, yo decidí regresar a México, estaba decepcionado del amor, muy lastimado por el divorcio y no 
me acostumbraba del todo a la vida en Norteamérica. En Estados Unidos me casé con una chava israelí, pero no duró nada mi matrimonio, a los dos años y medio ya estaba divorciado, ella de regreso en Israel y yo de nuevo en México. Solo y desempleado.

En el 2006, recién llegado de Boston, me invitaron al Iteso a sustituir a un maestro en la materia de Historia del Arte, de la carrera de Diseño Gráfico. Yo había trabajado mucho en el diseño y en edición de revistas y libros, me había vuelto un especialista. Tenía mucho para dar, bueno, así lo sentí en aquel entonces. El que me invitaran a incorporarme al Iteso, aunque fuera al inicio como profesor sustituto, me pareció toda una revelación, porque era mi casa de estudios, mi alma máter. Le tenía mucho cariño, bueno, aún le tengo. En él viví los mejores momentos de mi juventud. Además en aquel entonces acababa de regresar y no tenía como muchas opciones que digamos. Tuve mucha ilusión los primeros años de trabajar como maestro, pero luego los he ido perdiendo, la institución ayuda bastante.

Pues mi primer semestre fue todo un éxito, los alumnos quedaron muy contentos con mi trabajo y al siguiente ya estaba yo dando materias no solo en Diseño, sino también en Arquitectura y en Ciencias de la Comunicación. De todos modos freelanceaba todo lo que podía: hacía páginas webs, traducciones, daba clases particulares de inglés, hacía también chamba de diseño gráfico, construía la imagen de negocios, empresas, de lo que me cayera. No me iba mal de inicio.

Actualmente la consultoría ha bajado muchísimo, sobre todo porque para todo te piden facturas y recibos de honorarios, y pagos de impuestos. Ya no queda mucho o lo tienes que cobrar barato para que las empresas accedan.

Los primeros semestres estuve bien contento en el Iteso, bien ganoso de compartir mis experiencias con los chavos y ponerlos a reflexionar, pero con el paso del tiempo me fui dando cuenta de más cosas. No es lo mismo ser alumno; cuando eres estudiante el 
Iteso es de los lugares más bonitos del mundo, con sus áreas verdes, sus árboles, su biblioteca, los amigos, las chavas... Pero ya como profesor, las cosas son bien diferentes, bien cabronas, bien injustas. La verdad es que han ido empeorando y poniéndose cada vez más feas con el paso de los semestres.

Para empezar, te dicen hasta el último momento cuáles serán las materias que vas a dar al inicio del semestre. A veces hasta un día antes es que sabes qué materias vas a dar. Así no puedes hacer compromisos de trabajo con otra escuela, o agarrar algo más grande de freelance: una consultoría, una asesoría, nada... Puras cosas pequeñas. Realmente eso te atora tu desarrollo. Si tienes rato ya dando una clase, por ejemplo, consiguiendo materiales, trabajándole en la planeación, etcétera, nunca sabes si de repente le van a dar tu materia a alguien más, aunque hayas invertido muchísimo tiempo e incluso dinero en capacitarte y tener al tiro tu curso. De un momento a otro se la pueden pasar a una persona nueva que llegó, un amigo de algún coordinador, alguien con más influencia. O quizá a los alumnos ya no les cuadraste, a alguien no le pareció lo que le dijiste o cómo lo viste y te quedas fuera. Hay historias bien duras y bien tristes de compañeros que los hicieron irse sin la menor explicación, por las situaciones más inexplicables, porque a algún alumno no le gustó algo. Que de repente llegaban normalmente a su clase a trabajar y sin ninguna razón ya había otra persona impartiendo su clase sin que nadie les avisara ni les advirtiera. Los coordinadores no querían darles la cara, ¿por qué los corrían de esa manera, sin previo aviso, no más metiendo a alguien así, a la mala? Las peores injusticias las he visto en los últimos años aquí.

El arquitecto Gamaliel comenzó a sentirse crecientemente menos identificado con el Iteso en los últimos dos años, conforme la competencia entre profesores de asignatura también aumentó, lo que hizo que se presentaran cada vez situaciones más injustas e incómodas.

En este último año, descubrió también la acupuntura y el reiki, y su vocación por las medicinas alternativas, las cuales estudia los sábados. Esto le permitió darse un respiro fuera del Iteso: 
Al mismo tiempo que daba clases en el Iteso, empecé a estudiar un diplomado en terapias naturales. Lo encontré en un momento justo en que necesitaba una fuga fuera del trabajo en la universidad. Me había lesionado un tobillo en la bicicleta y una homeópata me curó muy rápido. La doctora vivía una vida bien relajada, no más atendiendo a sus pacientes, estudiando. Me di cuenta de que yo quería también una vida así. Me inscribí en una escuela y comencé a estudiar, a ver mis primeros pacientes y hacer planes para tener una vida cada vez más relajada y más independiente de cualquier institución.

Y, fíjate, justo cuando ya solo me quedaba una materia por impartir en el Iteso, cuando ya creía que la causa en esa escuela estaba perdida, justamente este semestre, me dieron más materias. Así es la cosa, nunca sabes. De repente un día te echan y no vuelves a saber nunca más del Iteso, pues no tienes ningún tipo de seguridad ahí. Y tienes que trabajar el duelo emocional, porque es muy duro separarte de una institución a la que quisiste mucho y que luego te puede dar una patada en el trasero. Mucha gente ha sufrido mucho porque la echaron del Iteso de muy fea manera.

Pero de repente pueden darte más chamba. Así es el Iteso de incomprensible, como el Universo.

\section{Profesores de asignatura}

Las universidades públicas en México también han seguido, paulatinamente y con cierta discreción, el paso hacia la contratación de profesores por hora. Aunque en ese esquema se les conoces como profesores de asignatura. Algunas escuelas privadas también utilizan el término de asignatura en lugar de tiempo variable; sin embargo, en esencia, son lo mismo, o casi lo mismo.

Desde el punto de vista fiscal y administrativo, tanto a los profesores de asignatura como a los de tiempo variable se les paga exclusivamente su hora de trabajo directa en el salón de clases. Todo el resto de actividades extracurriculares, las cuales cada día son mayores en 
las instituciones (planeación, llenado de formatos, juntas y reuniones de trabajo, cursos y capacitaciones), deberán correr por parte del docente, independientemente de si su sueldo sea suficiente o no para cubrir los gastos generados por las últimas. Los periodos vacacionales y sobre todo los días feriados, o de puente, como se dice en México, cuando se celebra alguna festividad nacional y no hay clase obviamente tampoco se les pagan.

Incluso, en frecuentes ocasiones, particularmente en las escuelas privadas, los docentes son obligados a firmar su renuncia junto con el contrato por horas. Renuncian de antemano para evitar que puedan demandar a la empresa en caso de alguna inconformidad y tampoco generarán ningún tipo de antigüedad ni prestación laboral.

Algunas escuelas y universidades sí pagan el diseño de cursos y las planeaciones, como la Universidad del Valle de México, pero la realidad es que es una muy rara excepción. Por lo menos esa noticia teníamos hasta inicios de 2015, pues la inmensa mayoría de las instituciones educativas en México dejan caer una inmensa carga de trabajo sobre los hombros de los profesores llamados de asignatura o tiempo variable, sin que esta labor resulte remunerada. Como se ha dicho, son largas las filas de profesionales aguardando por una oportunidad para impartir clases, al costo que sea, debido a la falta de empleos; por ello, las empresas educativas se dan el lujo de casi jugar con sus necesidades.

La maestra Amy tiene un largo background de experiencia docente y laboral en la Universidad de Guadalajara. Es licenciada en Historia, con una maestría en Educación, y comenzó a trabajar en la Universidad desde que era estudiante, como asistente de un profesor extranjero, quien desde entonces era miembro del Sistema Nacional de Investigadores. Su primer sueldo provino de las Becas para Estudiantes Sobresalientes, un programa institucional que en algunas ocasiones tenía el apoyo de la Secretaría de Educación Pública para brindar una pequeña beca por la ínfima cantidad de 400 pesos al mes, a los estudiantes cuyo promedio general fuera arriba de 9,5. Y en otras, corría a cargo de las arcas de la misma Universidad. A ella le resultaba de una ayuda excepcional, pues sus padres eran de escasos recursos. Cuando comenzó a recibir aquel pago, ese dinero le servía bastante para costear 
su transportación, desayunos, incluso lo estiraba para ayudar financieramente un poco a sus padres y a su novio:

Desde la prepa me gustaban mucho las ciencias sociales, las humanidades, la antropología, la arqueología y la historia. Yo estudié en la Escuela Preparatoria Vocacional, en la época en que todavía existía lo que se llamaban las áreas en la Universidad de Guadalajara. Los viernes y sábados tenías que dejar tu escuela para ir a otra, de la misma Universidad, en donde tomaba materias relacionadas con mi perfil. Era muy padre porque se compartían las clases con chavos de todas las prepas de Guadalajara, yo tuve mi primer novio en las asignaturas y luego conocí también ahí al que fue mi esposo. Toda la semana esperaba que se llegara el viernes porque me gustaban mucho las materias que llevaba y porque vería a mis amigos. Era una oportunidad para conocer tanto a compañeros con intereses y gustos más semejantes a los tuyos, como también a profesores que daban materias muy interesantes, más encaminadas a lo que ibas a estudiar.

En las clases de filosofía conocí a Gabriel, el que iba a ser el gran amor de mi vida y mi esposo durante ocho años.

Al terminar la prepa, estaba decidida a que iba a ser arqueóloga, mis profesores me animaban a que me fuera a la Ciudad de México a estudiar al Instituto Nacional de Antropología e Historia, pues ahí tienen la carrera de Arqueología. En mis tiempos en la Universidad de Guadalajara no había esa carrera ni siquiera Antropología, que era una segunda opción. Como mínimo existía la posibilidad de que me fuera a Zacatecas, a la Universidad Autónoma para estudiar, porque ahí sí tienen la carrera de Arqueología, muy ligada al trabajo de campo. Y la verdad es que la posibilidad de Zacatecas me atraía más que ninguna otra, porque está relativamente cerca de Guadalajara y porque es una ciudad muy bonita, colonial y muy tranquila.

Pero Gabriel, que en ese entonces era mi novio, no se quiso mover, él salió en listas en la carrera de Geografía casi de inmediato. 
También me la pensaba por mis papás, que ya eran grandes y no era fácil dejarlos. Tenía muchas ataduras que me retenían aquí en Guadalajara, así es que en aquel entonces me decidí por la opción de estudiar historia aquí en la U de G.

Era un tiempo muy bonito, mi tesis de licenciatura ganó un premio de excelencia y salió publicada. Gabriel tenía un pequeño restaurante, no le iba nada mal. Los dos estudiábamos por la tarde en la Facultad de Humanidades del CUCSH, nos veíamos en los recesos. Salimos embarazados desde la mitad de la carrera y de inicio Gabriel estuvo bien comprometido como papá. Estábamos muy contentos los primeros años.

Y pues las cosas comenzaron a cambiar, como siempre ocurre. Su negocio entró en bancarrota y él comenzó a dar clases por completo en diferentes prepas y escuelas. Lo notaba cada vez más individualista, solamente sus cosas, solo lo suyo, en eso llegó nuestro segundo bebé. Y él nada más metido en sus rollos, en sus lecturas, con sus cuates. Nunca quiso dejar la fiesta, aunque ya estaba grande y teníamos niños.

Mis papás me cuidaban a los niños, de inicio comencé trabajando en el Archivo Histórico de Zapopan. No era precisamente lo mío, pero había la posibilidad de conseguir una plaza.

Entonces se dio la posibilidad de ingresar a dar clases a una prepa de la U de G. Y pues, erróneamente, dejé el archivo. Yo todavía tenía mucho idealismo, pensaba que iba a ser fácil convertirme en investigadora de la Universidad como algunos de mis maestros. No conocía nada de los escalafones ni de los procesos administrativos de la U de G. De haberlo sabido no habría renunciado al Archivo de Zapopan. La verdad es que fui demasiado ingenua e idealista.

Ya estando al interior de las prepas de la U de G, te das cuenta que es toda una mafia. De entrada ves pasar a la gente por encima de ti, sobre todo a los que tienen influencias o pertenecen a los grupos políticos que controlan las prepas. Primero me exigían 
estar titulada de la maestría para poder acceder a una plaza de tiempo completo. Así, con todo y los niños chiquitos, me aventé el tiro y comencé a estudiarla en la SEP. Estaba bien segura y convencida de que iba a ser muy sencillo una vez con mi cédula de la maestría, conseguir mi base en la prepa. Casi al finalizar la maestría mi esposo se metió más y más en sus rollos, y pues nos separamos. Siempre tuve la esperanza de que sólo fuera parte de sus inmadureces, algo temporal. Pero nunca hubo reconciliación.

Fue bien difícil enfrentarme sola con los niños, titularme de la maestría, asimilar la separación de Gabriel.

Y luego resultó que la base en la U de G no llegó jamás, participé en dos convocatorias y hasta ahora no he tenido la suerte. Actualmente tengo quince años de antigüedad en la $U$ de $G$, y de repente me doy cuenta que sí se otorgan tiempos completos a las personas de confianza de los directores de prepa, o de más arriba. Pero si no estás con nadie políticamente hablando, nunca prosperarás.

Y está bien duro porque tengo dos niños aún chiquitos. Mis papás ya están muy grandes como para cuidarlos, y ni para qué te digo que tampoco recibo nada de ayuda de Gabriel... Yo tengo que sustentarlos económicamente a todos...

El esquema de profesor de asignatura al interior de la Universidad de Guadalajara hace que los profesores reciban un pago por materia que oscila entre los 60 u 80 pesos, dependiendo de la categoría y tiempo de antigüedad con que cuenten. En este caso, dependiendo de su antigüedad, pese al bajo costo de su salario, cuentan con ciertas prestaciones, como el pago de vacaciones, diversos beneficios de los organismos afiliados a la Universidad de Guadalajara: seguro social, becas para estudiar inglés, maestrías, etc., pero el salario continúa siendo de cualquier manera demasiado bajo. A tal grado que para una profesora como Amy resulta verdaderamente muy difícil sobrevivir económicamente y sostener a sus hijos y a sus padres con su salario. 


\section{La búsqueda de la independencia}

Este tipo de esquemas laborales en donde los sueldos como docentes de asignatura, por materia, como en el caso de Amy, son muy bajos y con pocas posibilidades de crecimiento o, como en el caso de Gamaliel, en donde las vacaciones no son pagadas y no cuentan con la más mínima seguridad laboral, obligan a que los docentes cada vez volteen hacia dentro de sí mismos en busca de alternativas por completo fuera de sus instituciones de trabajo, es el caso de Gamaliel, por ejemplo, quien planea fundar una clínica de terapias alternativas y no ve al Iteso de ningún modo, aunque lo quiere, como parte de su futuro.

La maestra Amy se encuentra en estos momentos estudiando en sus tiempos libres una pequeña carrera de corte y confección:

Yo estoy superdecepcionada de la Universidad, por eso me metí al DIF a estudiar costura. Todo esto a mí me ha enseñado que mi vida no se reduce al rollo académico. Yo creo que todos somos muy múltiples, muy versátiles, pero a veces nos limitamos a una sola opción.

Estoy aprendiendo a hacer blusas y vestidos de manta, y ropa para niños también de manta. Pienso empezar a diseñar ropa y a venderla por internet. A lo mejor la Universidad de Guadalajara sí es realmente un proyecto de vida cuando estás dentro de un grupo político o cuando ya se tiene la suerte de tener plaza. Pero yo nunca fui muy buena para la política, a mí me sigue gustando leer e investigar, y lo sigo haciendo. Ahorita estoy leyendo Las palabras y las cosas de Michel Foucault. Pero ya lo hago por mero placer, por el mero disfrute de la lectura porque ese autor siempre me gustó mucho, también era historiador como yo.

Pero sí, yo no me siento parte de la Universidad. Sin embargo mis niños ya están bien grandes y somos bien unidos, como que ellos me entienden mucho por todas las cosas que hemos tenido que pasar. Ya hice mi primer blusa y espero pronto comenzar a comercializarlas. En el municipio de Tlaquepaque tomé un curso 
de emprendimiento y nos asesoraron bien para poder comenzar un negocio. Calculo que no tendré que invertir mucho y vas a ver, pronto tendré mi propia tienda... 
